\title{
Brain MRI Findings in Pediatric-Onset Neuromyelitis Optica Spectrum Disorder: Challenges in Differentiation from Acute Disseminated Encephalomyelitis
}

\author{
(DE. Bulut, (D). Karakaya, DS. Salama, (D) M. Levy, (D)T.A.G.M. Huisman, and (DI. Izbudak
}

\begin{abstract}
BACKGROUND AND PURPOSE: Differentiating pediatric-onset neuromyelitis optica spectrum disorder from acute disseminated encephalomyelitis could be challenging, especially in cases presenting with only brain manifestations. Our purpose was to investigate brain MR imaging features that may help distinguish these 2 entities.
\end{abstract}

MATERIALS AND METHODS: We retrospectively examined initial brain MR imaging studies of 10 patients with pediatric-onset neuromyelitis optica spectrum disorder (female/male ratio, 7:3) and 10 patients with acute disseminated encephalomyelitis (female/male ratio, 2:8). The mean age of the patients was $10.3 \pm 5.6$ and $8.7 \pm 5.3$ years, respectively. Brain lesions were evaluated with respect to location, extent, expansion, $\mathrm{T} 1$ hypointensity, contrast enhancement/pattern, and diffusion characteristics. The $\chi^{2}$ test (Yates or Fisher exact $\chi^{2}$ tests) was used to compare differences between groups.

RESULTS: Cerebral subcortical \pm juxtacortical and pons \pm middle cerebellar peduncle were the most frequent locations involved in both neuromyelitis optica spectrum disorder ( $n=5$ and 4, respectively) and acute disseminated encephalomyelitis ( $n=9$ and 7, respectively). Thalamic lesions were more frequent in acute disseminated encephalomyelitis $(P=.020)$ and were detected only in 1 patient with neuromyelitis optica spectrum disorder. None of the patients with neuromyelitis optica spectrum disorder had hypothalamic, internal capsule, or cortical lesions. The internal capsule involvement was found to be significantly different between groups $(P=.033)$. There was no significant difference in terms of extent, expansion, $\mathrm{T} 7$ hypointensity, contrast enhancement/pattern, and diffusion characteristics.

CONCLUSIONS: Although there is a considerable overlap in brain MR imaging findings, thalamic and internal capsule involvement could be used to differentiate pediatric-onset neuromyelitis optica spectrum disorder from acute disseminated encephalomyelitis.

ABBREVIATIONS: ADEM = acute disseminated encephalomyelitis; anti-AQP4 = anti-aquaporin-4; $\mathrm{AQP4}$ = aquaporin-4; NMOSD = neuromyelitis optica spectrum disorder

$\mathbf{N}$ euromyelitis optica spectrum disorder (NMOSD) is a distinct inflammatory CNS disease characterized primarily by recurrent attacks of optic neuritis and/or longitudinally extensive transverse myelitis. The discovery of a highly specific serum

Received December 11, 2018; accepted after revision February 2, 2019.

From the Departments of Radiology (E.B.) and Statistics (J.K.), Hacettepe University Faculty of Medicine, Ankara, Turkey; Department of Neurology and Psychiatry (S.S.), University of Alexandria, Alexandria, Egypt; Department of Neurology (M.L.), Johns Hopkins School of Medicine, Baltimore, Maryland; Section of Pediatric Neuroradiology (I.I.), Division of Neuroradiology, The Russell H. Morgan Department of Radiology and Radiological Science, Johns Hopkins School of Medicine, Baltimore, Maryland; and Edward B. Singleton Chair of Radiology (T.A.G.M.H.), Texas Children's Hospital, Houston, Texas.

Paper previously presented, in part, as an oral presentation at: Annual Meeting of the American Society of Neuroradiology and the Foundation of the ASNR Symposium, June 2-7, 2018; Vancouver, British Columbia, Canada. The presentation was No. O-246.

Please address correspondence to Elif Bulut, MD, Department of Radiology, Hacettepe University Faculty of Medicine, Sıhhıye, 06230 Ankara, Turkey; e-mail: drelifbulut@yahoo.com

http://dx.doi.org/10.3174/ajnr.A6003 marker for neuromyelitis optica, the anti-aquaporin-4 (antiAQP4) antibody, provided increased recognition of previously precluded brain involvement ${ }^{1,2}$ and further expanded the clinical spectrum by identification of seropositive patients with atypical presentations. ${ }^{3,4}$

Previous studies in NMOSD defined the brain MR imaging lesions mostly as "nonspecific," located in areas of high or low aquaporin-4 (AQP4) expression. ${ }^{5,6}$ More recent characterizations of brain MR imaging abnormalities reported in seropositive patients include periependymal lesions surrounding the ventricles and cerebral aqueduct, dorsal medullary lesions often continuous with cervical cord lesions, extensive hemispheric white matter lesions, and lesions in the corticospinal tracts (posterior limb of the internal capsule and cerebral peduncle). ${ }^{7-10}$

Relative to adult-onset, NMOSD is infrequent in children ( $3 \%-5 \%$ of all cases). In series of pediatric-onset NMOSD, brain lesions are reported more frequently ${ }^{11-14}$; this feature makes the task of distinguishing the clinical picture of pediatric-onset 
NMOSD from other acquired demyelinating syndromes, such as acute disseminated encephalomyelitis (ADEM), very challenging. This is especially difficult in cases presenting with brain manifestations without optic neuritis or myelitis at onset. ${ }^{15,16}$ A timely and accurate diagnosis is important for effective management of NMOSD, which has a very different treatment approach compared with ADEM and other demyelinating diseases. ${ }^{17,18}$

The aim of our study was to identify brain MR imaging findings at disease onset that could help distinguish pediatric-onset NMOSD from ADEM.

\section{MATERIALS AND METHODS Patient Selection}

We obtained approval from the Johns Hopkins institutional review board to conduct a retrospective search of pediatric patients diagnosed with neuromyelitis optica or NMOSD between 2002 and 2017 at the Johns Hopkins Hospital. Inclusion criteria were as follows: pediatric onset (18 years or younger); fulfillment of the 2006 and 2015 criteria for neuromyelitis optica and NMOSD, respectively ${ }^{2,3}$; and cerebral manifestations at disease onset with technically available brain MRIs for review. The results of antiAQP4 antibody tests were also recorded.

For the ADEM group, the Johns Hopkins Hospital radiologic data base was queried for pediatric-onset ADEM. Inclusion criteria were as follows: patients fulfilling the 2007 consensus definition for ADEM, ${ }^{19}$ brain MR imaging studies obtained within 2 months of the first clinical event, and a follow-up time of at least 2 years. We excluded patients with further attacks that could suggest an alternative diagnosis.

\section{Image Acquisition}

All MR images were obtained using either 1.5T or 3T scanners (Achieva, Philips Healthcare, Best, the Netherlands; Signa Excite, GE Healthcare, Milwaukee, Wisconsin; and Area, Avanto, Skyra, Siemens, Erlangen, Germany). Brain MR imaging sequences were T1WI, fast spin-echo T2WI, fast spin-echo FLAIR, and postgadolinium T1-weighted imaging. DWI was also included except for 2 children with NMOSD. The T1WI was performed with the following parameters: TR range, 520-696 ms; TE range, 4.6-14 ms; matrix size range, $192 \times 192$ to $512 \times 196$; FOV range, $190 \times 190$ to $240 \times 240 \mathrm{~mm}$; section thickness/spacing range, $1 / 1$ to $5 / 7 \mathrm{~mm}$. The T2WI was performed with the following parameters: TR range, 2500-7000 ms; TE range, 83-112 ms; matrix size range, $256 \times 184$ to $448 \times 335$; FOV range, $159 \times 200$ to $240 \times 240 \mathrm{~mm}$; section thickness/spacing range, $2 / 2$ to $5 / 5 \mathrm{~mm}$. The axial FLAIR sequence was performed with the following parameters: TR range, 6000-9000 ms; TE range, $81-120 \mathrm{~ms}$; TI range, 2000-2600 ms; matrix size range, $256 \times 256$ to $416 \times 512$; FOV range, $159 \times$ 200 to $240 \times 240 \mathrm{~mm}$; section thickness range, $4-5 \mathrm{~mm}$. The axial EPI DWI was performed with the following parameters: TR range, 5400-9000 ms; TE range, 70-100 ms; matrix size range, $128 \times$ 128 to $192 \times 192$; FOV range, $300 \times 230$ to $350 \times 260 \mathrm{~mm}$; section thickness range, $4-5 \mathrm{~mm}$.

\section{Image Evaluation}

All brain MRIs were reviewed in consensus by 2 neuroradiologists (I.I. and E.B.). Brain lesions were evaluated with respect to loca-
Table 1: The clinical features of patients with pediatric-onset NMOSD vs ADEM

\begin{tabular}{lcc}
\hline \multicolumn{1}{c}{ Characteristics } & NMOSD & ADEM \\
\hline F/M ratio & $7: 3$ & $2: 8$ \\
Mean age at initial manifestation (yr) & $10.3 \pm 5.6$ & $8.7 \pm 5.3$ \\
Involvement at initial manifestation & & \\
$n(\%)$ & & \\
Brain & $4(40 \%)$ & $5(50 \%)$ \\
Brain + spinal cord & $2(20 \%)$ & $5(50 \%)$ \\
Brain + optic nerve & $2(20 \%)$ & 0 \\
Brain + spinal cord + optic nerve & $2(20 \%)$ & 0 \\
Mean follow-up time $(y r)$ & 5.1 & 3.4 \\
\hline
\end{tabular}

tion, extent, expansion, T1 hypointensity, contrast enhancement/ pattern, and diffusion characteristics.

\section{Statistical Analysis}

Data analysis was performed with SPSS Statistics 23.0 (IBM, Armonk, New York). Descriptive statistics were expressed as mean \pm SD for age. The independent-samples $t$ test was performed to compare the means of the 2 groups for age. Categoric variables are presented as count and percentage. The $\chi^{2}$ test (Yates or Fisher exact $\chi^{2}$ tests) was used to compare differences between groups for all categoric variables. Results with $P$ values $<.05$ were considered statistically significant. Post hoc power analysis was performed to justify the sample size.

\section{RESULTS}

Ten patients with the diagnosis of NMOSD (female/male ratio, $7: 3$; mean age, $10.3 \pm 5.6$ years) and 10 patients with the diagnosis of ADEM (female/male ratio, 2:8; mean age, $8.7 \pm 5.3$ years) were included in the study. The clinical variables of the 2 cohorts are compared in Table 1 . There was no significant difference in terms of sex $(P=.07)$ or mean age $(P=.5)$ of the patients in the 2 groups. The patients with NMOSD presented either with cerebral manifestations alone $(40 \%)$ or in conjunction with optic neuritis and/or transverse myelitis (60\%). Two (20\%) of them were seropositive for $\mathrm{AQP} 4$, and 1 patient was found to be seropositive for the anti-myelin oligodendrocyte glycoprotein antibody a few months after onset.

A summary of the imaging findings of patients with NMOSD and ADEM is provided in Table 2. The most frequent locations of involvement were the cerebral hemispheric white matter and pons \pm middle cerebellar peduncle in both $\operatorname{NMOSD}(n=5$ and 4 , respectively) and ADEM ( $n=9$ and 7 , respectively). The periventricular area of the fourth ventricle was involved in 5 patients with NMOSD (50\%). The periependymal area appeared to be affected via extension of large lesions at the brain stem or cerebellum rather than being the center of involvement (Fig $1 A-C$ ). Periventricular extension of brain stem lesions $(n=6,60 \%)$ was also common in patients with ADEM.

The periaqueductal area $(n=1)$, thalamus $(n=1)$, basal ganglia $(n=1)$, and corpus callosum $(n=1)$ were rare lesion locations in patients with NMOSD. There were no lesions in the periependymal area around the third ventricle, in the posterior limb of the internal capsule, or in the cerebral cortex. The single thalamic lesion in the NMOSD cohort was small and nonspecific and distant from the ependymal lining of the third ventricle. In contrast to patients with NMOSD, unilateral/bilateral $(n=4 / 3)$ 
Table 2: Brain MRI findings of patients with pediatric-onset NMOSD vs ADEM

\begin{tabular}{|c|c|c|c|}
\hline Characteristics & $\begin{array}{c}\text { NMOSD } \\
(n=10)\end{array}$ & $\begin{array}{l}\text { ADEM } \\
(n=10)\end{array}$ & $\begin{array}{c}P \\
\text { Value }\end{array}$ \\
\hline \multicolumn{4}{|l|}{ Lesion localization/infratentorial } \\
\hline Medulla & $4(40 \%)$ & $2(20 \%)$ & 1 \\
\hline Area postrema & $2(20 \%)$ & $1(10 \%)$ & 1 \\
\hline Cerebellum & $2(20 \%)$ & $2(20 \%)$ & 1 \\
\hline Pons & $4(40 \%)$ & $7(70 \%)$ & .37 \\
\hline Middle cerebellar peduncle & $4(40 \%)$ & $6(60 \%)$ & .65 \\
\hline Periventricular & $5(50 \%)$ & $6(60 \%)$ & 1 \\
\hline Mesencephalon & $2(20 \%)$ & $4(40 \%)$ & .63 \\
\hline Periaqueductal & $1(10 \%)$ & $1(10 \%)$ & 1 \\
\hline \multicolumn{4}{|c|}{ Lesion localization/supratentorial } \\
\hline \multicolumn{4}{|l|}{ Diencephalon } \\
\hline Thalamus & $1(10 \%)$ & $7(70 \%)$ & $.02^{\mathrm{a}}$ \\
\hline Hypothalamus & 0 & $3(30 \%)$ & .21 \\
\hline Periventricular & 0 & $5(50 \%)$ & .37 \\
\hline Internal capsule & 0 & $5(50 \%)$ & $.03^{\mathrm{a}}$ \\
\hline Basal ganglia & $1(10 \%)$ & $3(30 \%)$ & .58 \\
\hline Corpus callosum & $1(10 \%)$ & $2(20 \%)$ & 1 \\
\hline \multicolumn{4}{|l|}{ Cerebral white matter } \\
\hline Periventricular & $3(30 \%)$ & $6(60 \%)$ & .37 \\
\hline Juxtacortical-subcortical & $5(50 \%)$ & $9(90 \%)$ & .57 \\
\hline Cerebral cortex & 0 & $3(30 \%)$ & .21 \\
\hline Size $(\geq 2 \mathrm{~cm})$ & $6(60 \%)$ & $8(80 \%)$ & .63 \\
\hline $\mathrm{T}$ hypointensity & $7(70 \%)$ & $6(60 \%)$ & 1 \\
\hline Expansion & $7(70 \%)$ & $8(80 \%)$ & 1 \\
\hline Contrast enhancement & $8(80 \%)$ & $5(50 \%)$ & .35 \\
\hline \multicolumn{4}{|l|}{ Enhancement pattern } \\
\hline Patchy, nodular & 5 & 2 & \\
\hline Diffuse & 0 & 3 & \\
\hline Leptomeningeal & 3 & 0 & \\
\hline Ring-incomplete ring & 1 & 1 & \\
\hline Perivenular & 1 & 0 & \\
\hline Restricted diffusion & $2^{b}$ & $3(30 \%)$ & 1 \\
\hline
\end{tabular}

thalamic and internal capsule involvement were common in patients with ADEM (Fig 2) and demonstrated a significant difference between these 2 groups $(P=.020$ and $P=.033$, respectively). The post hoc power values of $82 \%$ and $80 \%$ were calculated for the thalamic and internal capsule variables, respectively.

There was no significant difference in terms of extent, expansion, T1 hypointensity, contrast enhancement/pattern, and diffusion characteristics. Contrast enhancement was frequent in patients with NMOSD $(n=8)$, with the most common pattern described as cloudlike parenchymal enhancement $(n=5)$. We also detected regional cerebral or cerebellar leptomeningeal enhancement (Fig $1 D-F)$ in patients with NMOSD $(n=3)$ contrary to the ADEM group $(P=.23)$. There was no specific type of enhancement in patients with ADEM; most of the contrast-enhanced lesions showed mild diffuse enhancement $(n=3)$. Restricted diffusion was a rare finding in patients with both NMOSD $(n=2)$ and $\operatorname{ADEM}(n=3)$.

\section{DISCUSSION}

The most recent consensus criteria for the diagnosis of NMOSD make special mention of the difficulty in distinguishing pediatriconset NMOSD from ADEM. ${ }^{4}$ Pediatric ADEM may also present with longitudinally extensive transverse myelitis similar to NMOSD; therefore, myelitis is not a reliable predictor of NMOSD in chil- dren. ${ }^{4,20}$ Further complicating the issue, brain lesions are more frequent in children compared with adult-onset NMOSD. ${ }^{12-14,21,22}$ Imaging findings that can help distinguish these rare neuroimmunologic diseases may help to confirm a diagnosis in children. Accordingly, in this study, we aimed to find differences in brain MR imaging features that could distinguish pediatric-onset NMOSD from its primary mimicker, ADEM.

Pediatric-onset NMOSD-related brain involvement, similar to adult-onset disease, has been characterized by large $(>2 \mathrm{~cm})$ subcortical white matter lesions and lesions within the areas of high AQP4 expression such as periependymal areas of the fourth and third ventricles. ${ }^{12,14}$ Our results were similar to those of previous studies in regard to the high frequency of confluent lesions in the cerebral subcortical white matter and brain stem, though periventricular involvement was not distinctive. The periventricular areas of the fourth ventricle rather seemed to be secondarily involved by extension of confluent brain stem lesions. The bandlike circumventricular lesions with or without pencilthin ependymal enhancement, which are highly suggestive of an immune-mediated reaction against ependymal linings, were not detected. Therefore, periventricular involvement around the fourth ventricle was not specific enough to be distinguished from periventricular extension of large, confluent brain stem lesions frequently observed in the ADEM cohort. This finding along with the lack of previously described periependymal diencephalic lesions could be partially explained by a low anti-AQP4 seropositivity ratio in the NMOSD cohort. Also, this pattern of diencephalic involvement was not a common MR imaging finding, even in the largest study conducted on children with anti-AQP4 antibodies, ${ }^{12}$ and its absence may be due to the small number of cases in our study.

We have found that thalamic and internal capsule involvement could differentiate pediatric-onset NMOSD from ADEM. Thalamic involvement was infrequent and limited to a nonspecific lesion in 1 patient with NMOSD. This result was similar to those in the previous cohorts of pediatric-onset NMOSD, which noted frequencies of thalamic lesions ranging from none to $13 \% .^{12,13,18,20}$ On the other hand, thalamic involvement in the ADEM cohort was frequent and characteristic, with mostly asymmetric, large, and poorly marginated lesions as previously reported. ${ }^{23}$ The consistency of our findings with previous reports allowed us to postulate that thamic lesion location could favor a diagnosis of ADEM over pediatric-onset NMOSD.

Another lesion location that resulted in a significant difference between these disease entities was the posterior limb of the internal capsule. We did not detect any posterior limb of the internal capsule lesion in the NMOSD cohort, contrary to ADEM. Prior pediatric-onset NMOSD cohorts did not specify involvement of the internal capsule; therefore, a correlation with the current study is not possible. However, previous reports of adult-onset NMOSD showed varying frequencies of corticospinal tract involvement from $3 \%$ to $44 \% .^{5,7,10}$ The inconsistency of our findings with adult-onset NMOSD cohorts may reflect differences in disease mechanisms between children and adults. The statistical effects of small cohort size may also be responsible for this result. Re-evaluation with larger comparative cohorts is needed for validation. 


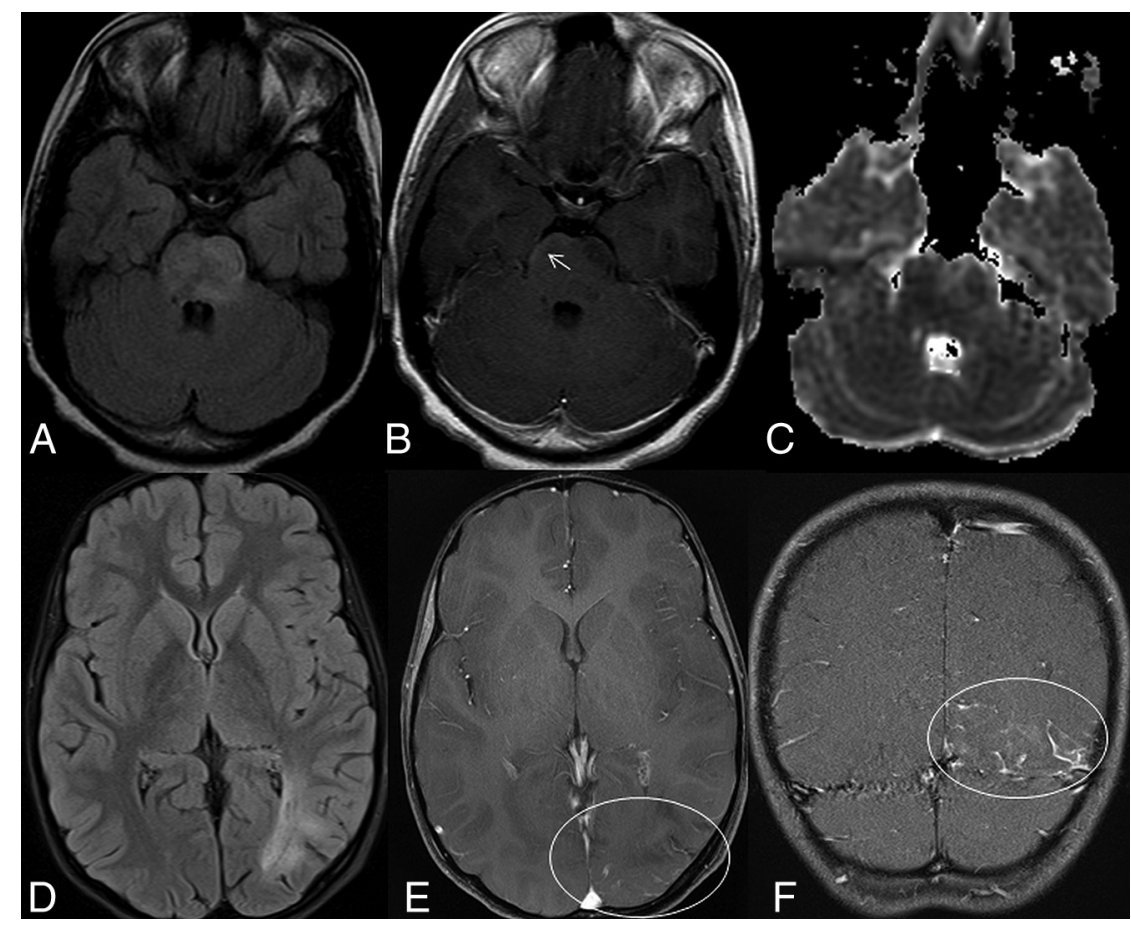

FIG 1. MR images of 2 patients in the NMOSD group. The axial FLAIR image ( $A$ ) of an 11-year-old female patient shows a minimally expansile, large, hyperintense lesion involving the pons. The postcontrast T-weighted image $(B)$ reveals inconspicuous subpial enhancement in the lesion (arrow). The patchy areas of restricted diffusion can be seen on the DWI $(C)$. The axial FLAIR image $(D)$ of another patient shows extensive white matter lesions in the left occipital lobe. The axial $(E)$ and the coronal $(F)$ postcontrast T1-weighted images reveal accompanying leptomeningeal enhancement (circles).

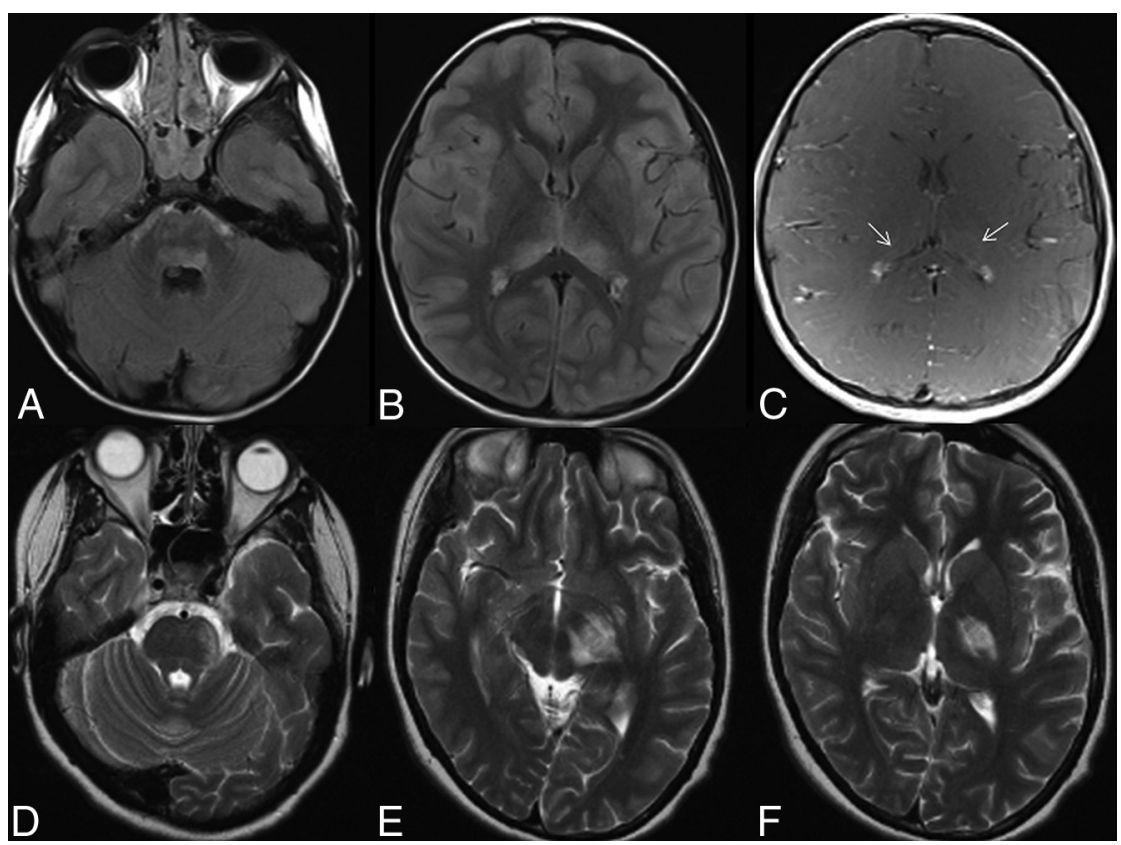

FIG 2. MR images of 2 patients in the ADEM group. The axial FLAIR images ( $A$ and $B$ ) of a 5 -year-old male patient show confluent pontine and symmetric thalamic lesions, respectively. The postcontrast T1-weighted image (C) reveals inconspicuous enhancement in the thalamic lesions (arrows). The axial T2-weighted images ( $D-F$ ) of a 16-year-old female patient show extensive lesions involving the pons, cerebral peduncle, and posterior limb of the internal capsule along the course of left corticospinal tract.

Remarkably, no other MR imaging findings including lesion expansion, T1 hypointensity, contrast enhancement/pattern, and diffusion characteristics could be identified to differentiate pediatric-onset NMOSD from ADEM. Expansion and T1 hypointensity are common features in the acute attack of demyelinating/inflammatory entities as contrast enhancement. ${ }^{23,24}$ Different patterns of contrast enhancement have been identified in NMOSD, such as cloudlike parenchymal, pencil-thin ependymal, leptomeningeal, or perivascular enhancement. ${ }^{24-28}$ Among these, ependymal and leptomeningeal enhancement was found significantly more frequently in patients with NMOSD than in those with MS and was suggested as a characteristic feature in NMOSD. ${ }^{27}$ In our study, we found similar results regarding leptomeningeal enhancement; it was seen in 3 patients $(37.5 \%)$ with NMOSD and was not detected in patients with $\operatorname{ADEM}(P=.23)$. Although differences between the 2 entities were not statistically significant, considering the previous remarks on leptomeningeal enhancement in NMOSD and the possible statistical effects of our smallsized cohorts together, we hypothesized that this enhancement pattern could favor the diagnosis of NMOSD over ADEM. Future investigations with larger comparative cohorts are required to confirm this hypothesis.

Two patients with leptomeningeal enhancement tested negative for antiAQP4 antibodies. This finding is difficult to explain by the anti-AQP4 antibodymediated immune response alone, which was the previously proposed mechanism for leptomeningeal enhancement. Fluctuating anti-AQ4 antibody titers, relatively lower antibody titers that are undetectable by serum assays, or varied AQP4 expression could be responsible for leptomeningeal enhancement in these seronegative patients, or perhaps some other underlying pathophysiologic mechanisms could exist. Although the patchy or so-called cloudlike enhancement was frequent in the NMOSD group, it was not discriminative and was also seen in patients with ADEM.

There are only a few case series in the literature defining diffusion characteristics of brain lesions in NMOSD. ${ }^{16,19,29}$ Brain lesions in those series were characterized by facilitated diffusion, which was attributed to vasogenic edema associated with acute inflammation. We present 2 NMOSD cases having brain lesions with patchy areas of restricted diffusion, 
different from previous data. Restricted diffusion in the affected regions may be due to swelling of myelin sheaths, reversible vascular compromise, or intense cell infiltration due to acute inflammatory reaction. Similar to findings in a previous report, ${ }^{30}$ restricted diffusion in the brain lesions was not frequent in our ADEM cohort, either. All these findings suggest that restricted diffusion could infrequently associate brain lesions in both pediatric-onset NMOSD and patients with ADEM and could not help in differentiating these 2 entities.

As the main limitation, our comparative cohort comprised a relatively small number of patients in each group. Therefore, future studies with larger sizes are needed to test the validity of the identified MR imaging discriminators and to further investigate the role of leptomeningeal enhancement in the differentiation of pediatric-onset NMOSD from ADEM.

Another limitation is that not all patients were tested for the anti-myelin oligodendrocyte glycoprotein antibody because testing for this antibody was not widely available at the time of the study design. Anti-myelin oligodendrocyte glycoprotein antibody has been found in a subgroup of anti AQP4 antibody negative NMOSD patients including children. Although anti-myelin oligodendrocyte glycoprotein antibody disease emerged as a distinct entity with more favorable clinical outcomes recently, ${ }^{31}$ it is not specified in the NMOSD diagnostic criteria yet. Larger cohorts with anti-myelin oligodendrocyte glycoprotein antibody testing are needed to characterize brain MR imaging findings distinctive of this group of patients in future studies.

Other limitations were due to retrospective design of the study. MR images were obtained from scanners with different magnet strengths, there were differences in imaging parameters including slice thickness, and DWI was not performed in 2 children with NMOSD. All of these could affect the sensitivity of image evaluation and comparisons.

\section{CONCLUSIONS}

Although there is a considerable overlap in brain MR imaging findings, the present study suggests that thalamic or internal capsule involvement could be used to differentiate pediatric-onset NMOSD from ADEM. Prospective studies with larger numbers of patients are required for validation of these findings.

Disclosures: Thierry A.G.M. Huisman-UNRELATED: Board Membership: American Journal of Neuroradiology Senior Editor. Izlem Izbudak-UNRELATED: Consultancy: Alexion Pharmaceuticals, Comments: reviewing MRI spine examinations of patients with neuromyelitis optica for adjudication about recurrence findings on MRI as part of an NMO trial; Grants/Grants Pending: Biogen, Comments: MS PATHS study site.* Sara Salama—RELATED: Grant: Ministry of Higher Education, Egypt, Comments: The Joint Supervision Scholarship number is JS-3725. Michael LevyUNRELATED: Consultancy: Alexion Pharmaceuticals, Quest Diagnostics; Expert Testimony: various law firms; Grants/Grants Pending: National Institutes of Health, National MS Society. * Money paid to the institution.

\section{REFERENCES}

1. Wingerchuk DM, Hogancamp WF, O’Brien PC, et al. The clinical course of neuromyelitis optica (Devic's syndrome). Neurology 1999; 53:1107-14 CrossRef Medline

2. Wingerchuk DM, Lennon VA, Pittock SJ, et al. Revised diagnostic criteria for neuromyelitis optica. Neurology 2006;66:1485-89 CrossRef Medline

3. Wingerchuk DM, Lennon VA, Lucchinetti CF, et al. The spectrum of neuromyelitis optica. Lancet Neurol 2007;6:805-15 CrossRef Medline

4. Wingerchuk DM, Banwell B, Bennett JL, et al; International Panel for NMO Diagnosis. International consensus diagnostic criteria for neuromyelitis optica spectrum disorders. Neurology 2015;85:177-89 CrossRef Medline

5. Pittock SJ, Lennon VA, Krecke K, et al. Brain abnormalities in neuromyelitis optica. Arch Neurol 2006;63:390-96 CrossRef Medline

6. Pittock SJ, Weinshenker BG, Lucchinetti CF, et al. Neuromyelitis optica brain lesions localized at sites of high aquaporin 4 expression. Arch Neurol 2006;63:964-68 CrossRef Medline

7. Kim W, Park MS, Lee SH, et al. Characteristic brain magnetic resonance imaging abnormalities in central nervous system aquaporin-4 autoimmunity. Mult Scler 2010;16:1229-36 CrossRef Medline

8. Kim HJ, Paul F, Lana-Peixoto MA, et al; Guthy-Jackson Charitable Foundation NMO International Clinical Consortium \& Biorepository. MRI characteristics of neuromyelitis optica spectrum disorder: an international update. Neurology 2015;84:1165-73 CrossRef Medline

9. Katz Sand I. Neuromyelitis optica spectrum disorders. Continuum (Minneap Minn) 2016;22:864-96 CrossRef Medline

10. Kim JE, Kim SM, Ahn SW, et al. Brain abnormalities in neuromyelitis optica. J Neurol Sci 2011;302:43-48 CrossRef Medline

11. Tenembaum S, Chitnis T, Nakashima I, et al. Neuromyelitis optica spectrum disorders in children and adolescents. Neurology 2016;87: S59-66 CrossRef Medline

12. McKeon A, Lennon VA, Lotze T, et al. CNS aquaporin-4 autoimmunity in children. Neurology 2008;71:93-100 CrossRef Medline

13. Absoud M, Lim MJ, Appleton R, et al. Paediatric neuromyelitis optica: clinical, MRI of the brain and prognostic features. J Neurol Neurosurg Psychiatry 2015;86:470-72 CrossRef Medline

14. Collongues N, Marignier R, Zéphir H, et al. Long-term follow-up of neuromyelitis optica with a pediatric onset. Neurology 2010;75: 1084-88 CrossRef Medline

15. Rostasy K, Bajer-Kornek B, Venkateswaran S, et al. Differential diagnosis and evaluation in pediatric inflammatory demyelinating disorders. Neurology 2016;87:S28-37 CrossRef Medline

16. Kim W, Kim SH, Lee $\mathrm{SH}$, et al. Brain abnormalities as an initial manifestation of neuromyelitis optica spectrum disorder. Mult Scler 2011;17:1107-12 CrossRef Medline

17. Wingerchuk DM, Weinshenker BG. Neuromyelitis optica. Curr Treat Options Neurol 2008;10:55-66 CrossRef Medline

18. Lotze TE, Northrop JL, Hutton GJ, et al. Spectrum of pediatric neuromyelitis optica. Pediatrics 2008;122:e1039-47 CrossRef Medline

19. Matsushita T, Isobe N, Matsuoka T, et al. Extensive vasogenic edema of anti-aquaporin-4 antibody-related brain lesions. Mult Scler 2009; 15:1113-17 CrossRef Medline

20. Banwell B, Tenembaum S, Lennon VA, et al. Neuromyelitis opticaIgG in childhood inflammatory demyelinating CNS disorders. Neurology 2008;70:344-52 CrossRef Medline

21. Kremer L, Mealy M, Jacob A, et al. Brainstem manifestations in neuromyelitis optica: a multicenter study of 258 patients. Mult Scler 2014;20:843-47 CrossRef Medline

22. Peña JA, Ravelo ME, Mora-La Cruz E, et al. NMO in pediatric patients: brain involvement and clinical expression. Arq Neuropsiquiatr 2011;69:34-38 CrossRef Medline

23. Marin SE, Callen DJ. The magnetic resonance imaging appearance of monophasic acute disseminated encephalomyelitis: an update post application of the 2007 consensus criteria. Neuroimaging Clin N Am 2013;23:245-66 CrossRef Medline

24. Orman G, Wang KY, Pekcevik Y, et al. Enhancing brain lesions during acute optic neuritis and/or longitudinally extensive transverse myelitis may portend a higher relapse rate in neuromyelitis optica spectrum disorders. AJNR Am J Neuroradiol 2017; 38:949-53 CrossRef Medline

25. Ito S, Mori M, Makino T, et al. “Cloud-like enhancement” is a magnetic resonance imaging abnormality specific to neuromyelitis optica. Ann Neurol 2009;66:425-28 CrossRef Medline

26. Banker P, Sonni S, Kister I, et al. Pencil-thin ependymal enhance- 
ment in neuromyelitis optica spectrum disorders. Mult Scler 2012; 18:1050-53 CrossRef Medline

27. Long $\mathrm{Y}$, Chen $\mathrm{M}$, Zhang B, et al. Brain gadolinium enhancement along the ventricular and leptomeningeal regions in patients with aquaporin-4 antibodies in cerebral spinal fluid. J Neuroimmunol 2014;269:62-67 CrossRef Medline

28. Pekcevik Y, Izbudak I. Perivascular enhancement in a patient with neuromyelitis optica spectrum disease during an optic neuritis attack. J Neuroimaging 2015;25:686-87 CrossRef Medline

29. Saiki S, Ueno Y, Moritani T, et al. Extensive hemispheric lesions with radiological evidence of blood-brain barrier integrity in a patient with neuromyelitis optica. J Neurol Sci 2009;284:217-19 CrossRef Medline

30. Zuccoli G, Panigrahy A, Sreedher G, et al. Vasogenic edema characterizes pediatric acute disseminated encephalomyelitis. Neuroradiology 2014;56:679-84 CrossRef Medline

31. Narayan R, Simpson A, Fritsche K, et al. MOG antibody disease: a review of MOG antibody seropositive neuromyelitis optica spectrum disorder. Mult Scler Relat Disord 2018;25:66-72 CrossRef Medline 